\title{
Cardiac Sarcoidosis Masquerading as Right Ventricular Dysplasia
}

\author{
PETER OTT, * FRANK I. MARCUS, * RICHARD E. SOBONYA, * FRED MORADY,† \\ BRADLEY P. KNIGHT,† and CHARLES E. FUENZALIDA‡ \\ From the *University of Arizona Health Sciences Center, Tucson, Arizona, +University of Michigan Health System, \\ Ann Arbor, Michigan, and the ¥Sarver Heart Center, Denver, Colorado
}

OTT, P., ET AL.: Cardiac Sarcoidosis Masquerading as Right Ventricular Dysplasia. Patients with cardiac sarcoidosis may present with clinical and morphological features similar to arrhythmogenic right ventricular dysplasia (ARVD) or cardiomyopathy (ARVC). Three cases of cardiac sarcoidosis are presented that clinically mimicked ARVD or ARVC until a pathology diagnosis of sarcoidosis was made at biopsy or autopsy. A diagnostic distinction, while often difficult to make, is important since treatment with corticosteroids may benefit those with sarcoidosis but is not expected to be useful in cases with ARVD or ARVC. (PACE 2003; 26[Pt. I]:1498-1503)

\section{sarcoidosis, arrhythmogenic right ventricular dysplasia/cardiomyopathy, ventricular tachycardia}

\section{Introduction}

Arrhythmogenic right ventricular cardiomyopathy (ARVC) or dysplasia (ARVD) is an inheritable heart muscle disease characterized by ventricular arrhythmias and structural abnormalities, predominantly of the right ventricle (RV). ${ }^{1}$ The RV myocardium undergoes progressive replacement with fibrous and fatty tissue. The disease has a predilection to affect the RV outflow tract, the RV apex, and the posterior subtricuspid region ("triangle of dysplasia"). In these regions there may be hypocontractility or even aneurysm formation. ARVC or ARVD is suspected in individuals who have ventricular arrhythmias, including premature ventricular beats, nonsustained or sustained ventricular tachycardia (VT), or arrhythmic sudden death, when there is generalized dilatation or localized abnormalities of the RV in the presence of preserved left ventricular size and function. However, the diagnosis can be difficult, particularly if there are only mild structural abnormalities in the RV.

It is not commonly appreciated that myocardial involvement in sarcoidosis can mimic some of the clinical and the structural abnormalities of ARVC or ARVD. This report presents three patients who were diagnosed clinically as having ARVC or ARVD but pathologically had myocardial sarcoidosis.

\section{Patient 1}

A previously healthy 37-year-old white man developed sudden shortness of breath and di-

Address for reprints: Peter Ott, M.D., Dept. of Cardiology, University of Arizona Health Sciences Center, 1501 N. Campbell Ave., Tucson, AZ 85724. Fax: (520) 626-4333; e-mail: ottp@u.arizona.edu

Received February 25, 2002; revised April 25, 2002, accepted October 4, 2002. aphoresis. His electrocardiogram showed a wide complex tachycardia (heart rate 200 beats/min) with a left bundle branch block morphology and right-axis deviation. Intravenous adenosine did not convert his arrhythmia, and he was treated with electric cardioversion. After conversion, his 12-lead electrocardiogram showed sinus rhythm (heart rate of 90 beats $/ \mathrm{min}$ ) with $\mathrm{T}$ wave inversion in leads $V_{1}$ and $V_{2}$ and a biphasic $T$ wave in lead $\mathrm{V}_{3}$ (Fig. 1).

He had no past medical history or family history of heart disease or sudden death. The patient reported a 2-3 week history of a nonproductive cough. A chest film after admission showed a normal heart size and diffuse interstitial alveolar opacities bilaterally, prominent in the perihilar and right basilar distribution. These findings were interpreted as consistent with pulmonary edema or possibly a superimposed pneumonia. All laboratory data were normal except for a moderately elevated lactate dehydrogenase (LDH). There was no evidence of an acute myocardial infarction. Cardiac catheterization showed normal coronary arteries without evidence of coronary artery spasm after ergonovine challenge, and his left ventricular ejection fraction was 0.74 .

A cardiac magnetic resonance imaging (MRI) was interpreted as showing areas of thinning of the RV free wall. There was no increase in signal intensity. During electrophysiological study, VT of two different morphologies, all with left bundle branch block configuration, was induced. He was diagnosed as having ARVC or ARVD. VT remained inducible after treatment with flecainide and atenolol but became noninducible on sotalol (160 mg bid).

Sotalol was gradually increased over the next 12 months to $320 \mathrm{mg}$ bid due to recurrent VT. Eventually sotalol was discontinued due to inefficacy and amiodarone was initiated. At that time a chest 


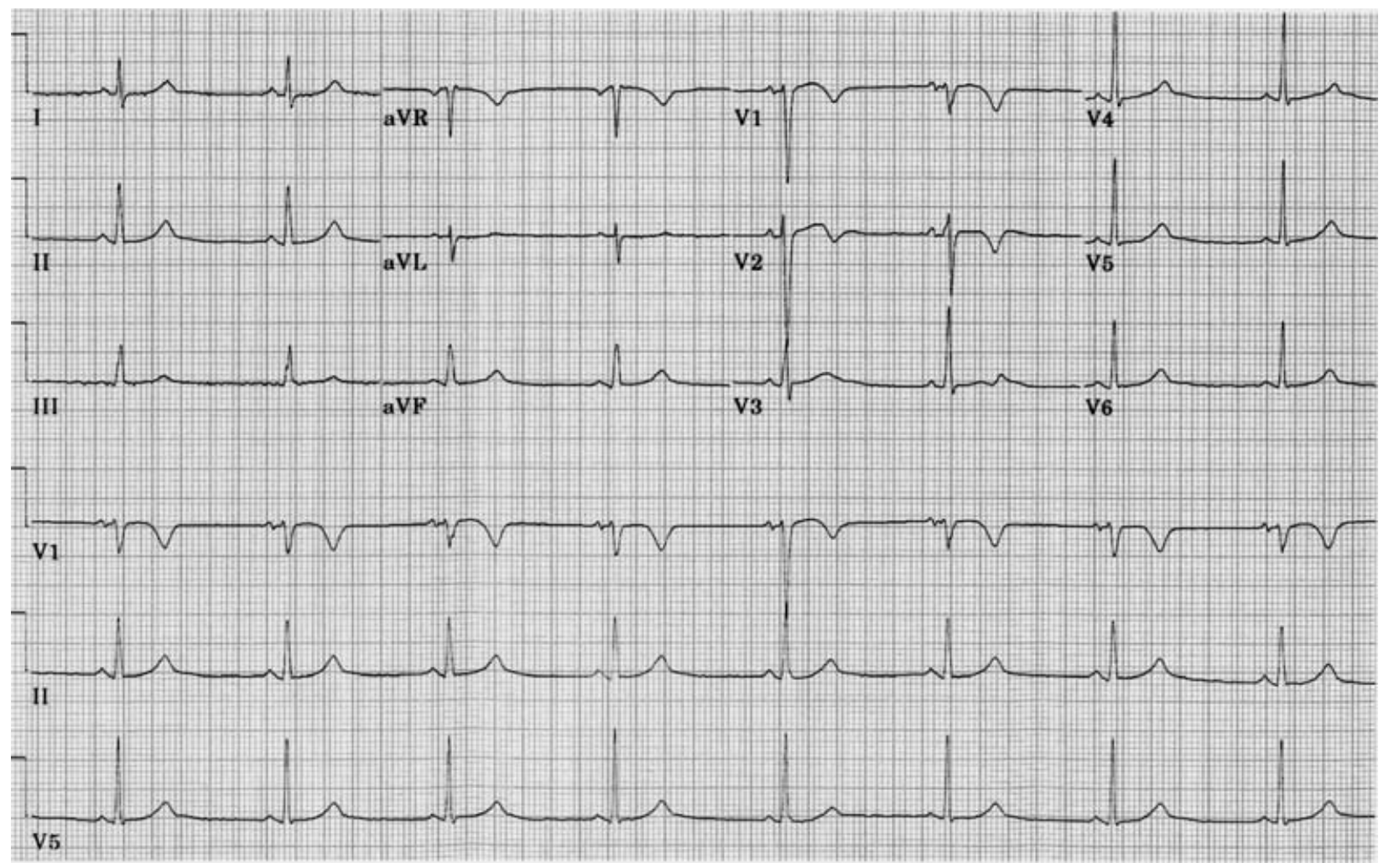

Figure 1. Twelve-lead surface electrocardiogram (1 mm/mV; $25 \mathrm{~cm} / \mathrm{s})$. The tracing shows abnormal T waves in leads $V_{1-3}$.

film showed a diffuse reticular-nodular pattern, particularly prominent in the upper lobes. A high resolution computed tomography (CT) scan confirmed these findings. Hilar adenopathy was not seen. A transbronchial biopsy was obtained that showed numerous noncaseating granulomas consistent with sarcoidosis.

The patient was then treated with prednisone. However, due to recurrent VT, an implantable cardioverter defibrillator (ICD) was implanted with excellent ventricular pacing and sensing thresholds. The patient improved on a combination therapy with amiodarone, atenolol, and immunosuppressive therapy and had only occasional episodes of VT treated successfully by his ICD.

Three years after the initial episode, an echocardiogram showed normal left ventricular systolic function, RV enlargement, and mild to moderately decreased RV systolic function. A chest film showed a right paratracheal mass and enlargement of the right hilum, all consistent with sarcoidosis.

\section{Comment}

This patient was diagnosed as having ARVC or ARVD on the basis of sustained VT of suspected RV origin, normal left ventricular function, and
RV structural changes compatible with ARVC or ARVD. Subtle pulmonary findings, present at the time of initial presentation, may have been due to pulmonary sarcoidosis, as confirmed later by histology. The diagnosis of cardiac sarcoidosis is made by inference.

\section{Patient 2}

A 47-year-old man without significant past medical history had the sudden onset of VT at the rate of 260 beats/min. The QRS morphology was that of left bundle branch block. This was treated with direct current (DC) cardioversion. His baseline electrocardiogram (Fig. 2) showed sinus rhythm with a PR interval of $220 \mathrm{~ms}$. The QRS duration was $110 \mathrm{~ms}$ with a pattern of incomplete right bundle branch block. A sharp deflection at the end of the QRS complex was interpreted as an epsilon wave.

An echocardiogram demonstrated significant RV enlargement (twice the size of the left ventricle) with a markedly global decrease in RV contractility. Cardiac catheterization showed normal coronary arteries. On angiography, there was severe general hypokinesis of the RV with mild tricuspid regurgitation. The left ventricle showed localized hypokinesis in the lateral and mid-inferior 


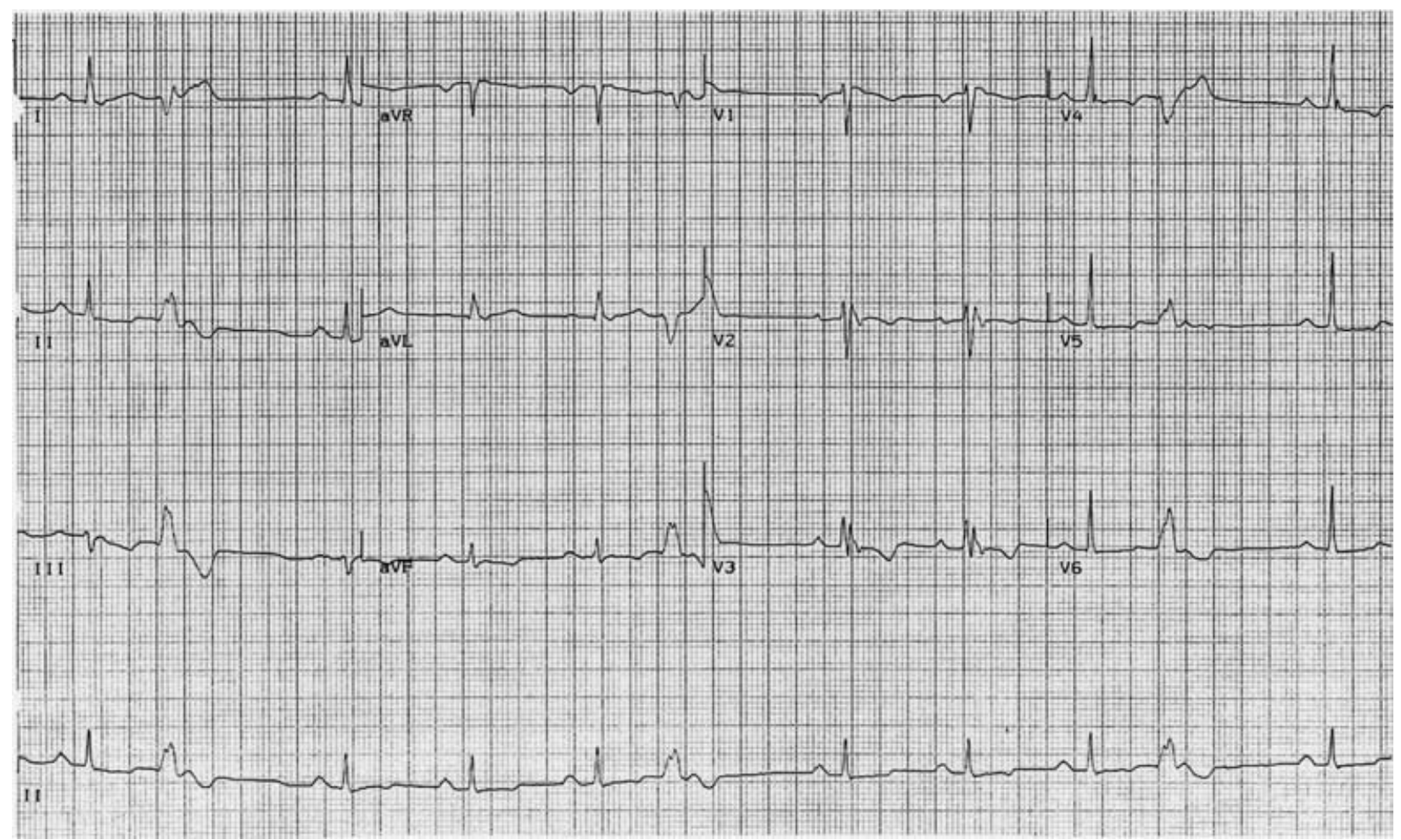

Figure 2. Twelve-lead surface electrocardiogram $(1 \mathrm{~mm} / \mathrm{mV} ; 25 \mathrm{~cm} / \mathrm{s})$. The tracing shows sinus rhythm with frequent premature ventricular contractions. The QRS complex in leads $V_{1-3}$ shows a rSr' morphology and the T waves are inverted in leads $V_{1-4}$.

wall, resulting in mild to moderate general hypokinesis and an estimated ejection fraction of 0.37. A cardiac MRI study was unsuccessful due to inadequate gating.

On electrophysiological study, VT (rate 250 beats/min) with a left bundle branch block morphology and inferior QRS axis was easily induced. The patient was diagnosed as having ARVC or ARVD. An ICD was implanted, and sotalol was prescribed.

A myocardial perfusion scan showed mild thinning in the inferior wall of the left ventricle compatible with a diaphragmatic infarction. Several RV septal myocardial biopsies were nonspecific showing interstitial edema and fibrosis, compatible with idiopathic congestive cardiomyopathy.

Over the next 2 years the patient had recurrence of VT, despite an increase in the dose of sotalol to $240 \mathrm{mg}$ bid In addition, he developed high degree atrioventricular (AV) block that persisted despite stopping sotalol. His ICD was revised to a dual chamber system. Flecainide controlled the VT, but resulted in loss of ventricular pacing capture necessitating the implantation of a new ventricular pacing lead. During the implant procedure $15 \mathrm{RV}$ sites were assessed and found to have in- adequate pacing thresholds. Finally, the lead was implanted at a RV apical site. Five days after discharge, the patient collapsed and could not be resuscitated.

Autopsy was restricted to the heart. The RV was markedly dilated with a free wall thickness of 1-4 mm (normal $3.8 \pm 0.9 \mathrm{~mm}$ ). The infundibulum and posterior free wall particularly showed thinning and fibrosis. The apex had increased trabeculation and was of normal thickness. The septum and left ventricle had several areas of subendocardial fibrosis. The left ventricular free wall averaged $1.5 \mathrm{~cm}$ in thickness. Microscopically, the myocardium showed nonnecrotizing granulomas of epitheloid and multinucleated giant cells surrounded by a rim of lymphocytes in a dense matrix of fibrous tissue (Fig. 3). Occasionally, giant cells had asteroid bodies, but no conchoid bodies were seen. There was no evidence of fibrofatty replacement of the myocardium. Adjacent lymph nodes showed similar granulomas. Stains for fungi and acid-fast bacilli were negative.

\section{Comment}

This patient presented with VT of suspected RV origin, an abnormal resting electrocardiograph, and severe RV enlargement that are features 


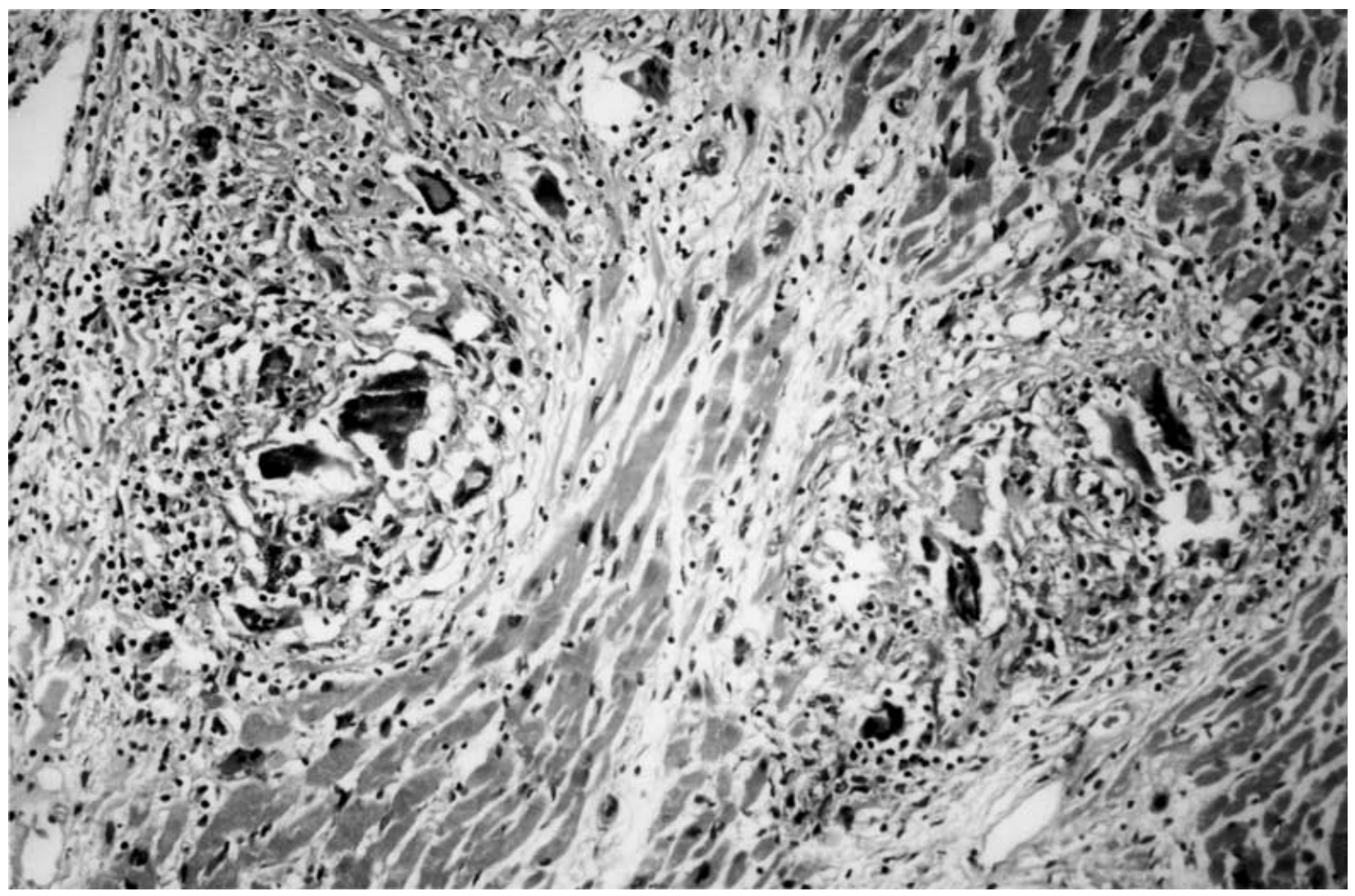

Figure 3. Two granulomas with giant cells in the myocardium. (Hematoxylin-Eosin, X530)

suggestive of ARVC or ARVD. At autopsy, he was shown to have sarcoidosis involving the myocardium and thoracic lymph nodes.

\section{Patient 3}

A 33-year-old man collapsed while playing racquetball. Extensive resuscitation efforts were unsuccessful. He had no past medical history, in particular there was no history of beryllium exposure, and he was not taking any medication and had no family history of cardiac disease or sudden death. An autopsy showed RV dilatation, initially suggesting possible ARVC or ARVD. However, microscopic exam showed considerable replacement of the right and left ventricle and interatrial septum by fibrosing but nonnecrotizing granulomas. Involvement of the RV was predominant. No background of lymphocytic myocarditis or scarring unrelated to granulomas was present, and no fibrofatty replacement of the myocardium was seen. Similar granulomas were present in the lungs and spleen. Histological stains for acid-fast organisms and fungi were negative.

\section{Comment}

ARVC or ARVD must be considered in the differential diagnosis of young adults dying suddenly during exercise. In the present case, RV enlargement, seen at gross pathology, seemed to support this diagnosis. Microscopic examination, however, revealed cardiac sarcoidosis.

\section{Discussion}

Sarcoidosis is a systemic granulomatous disorder of unknown etiology characterized by noncaseating epitheloid cell granulomas. It was named by Boeck, a Norwegian dermatologist, who in 1899 described nodular skin lesions of epitheloid cells. He thought that these cells resembled sarcoma cells; hence the designation "sarcoid."2

Sarcoidosis may involve practically every organ in the body. ${ }^{2}$ Cardiac involvement primarily documented at autopsy occurs in up to 20$50 \%$ of patients, most with disseminated sarcoidosis. $^{3,4}$ Cardiac pathology shows cardiac granulomas or areas of myocardial scarring with thinning and/or aneurysmal dilatation (Fig. 4). There is usually a preponderance of left ventricular and septal 


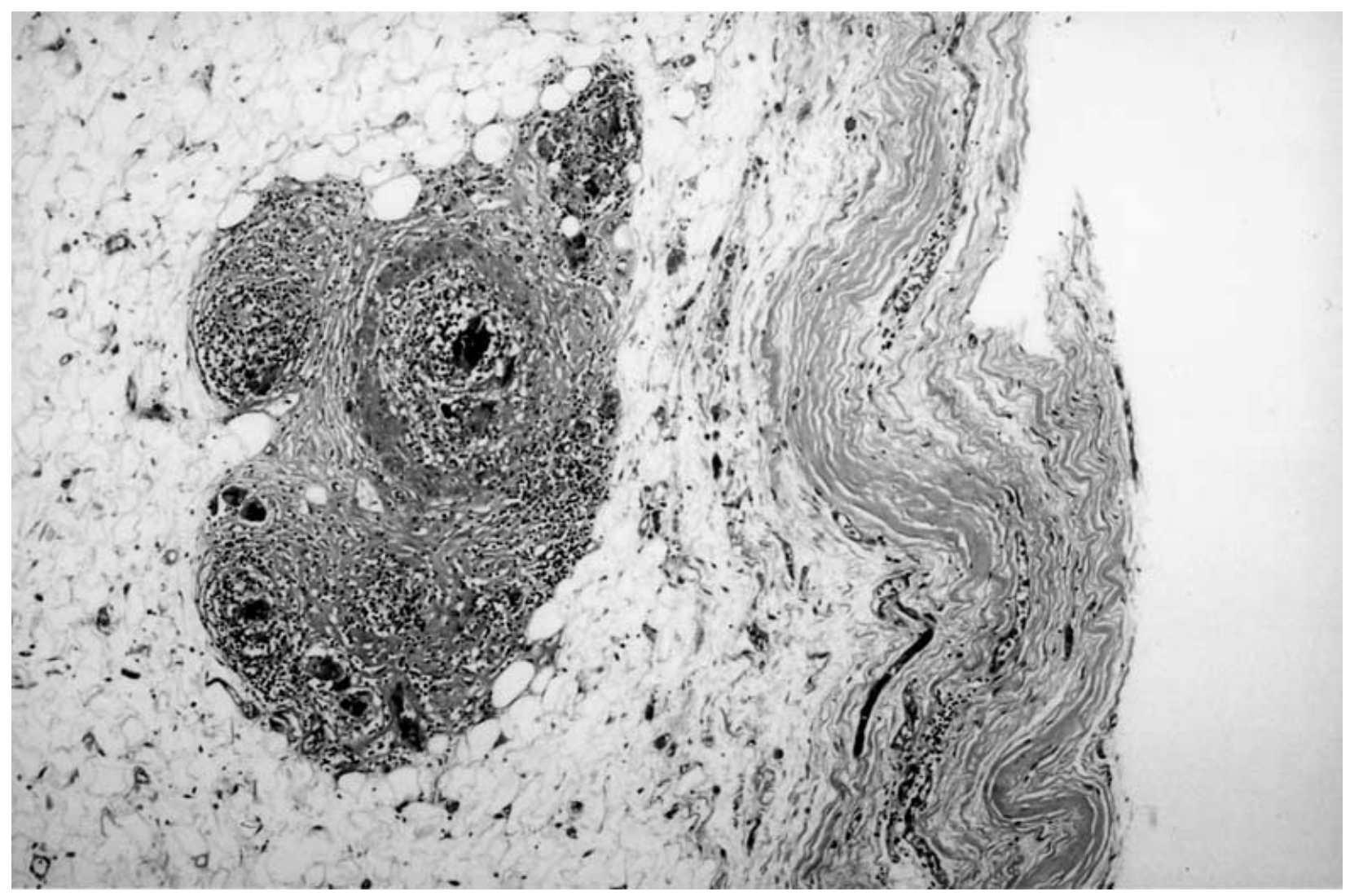

Figure 4. The right ventricular myocardium is reduced to a thin fibrous wall (right). The epicardial fat contains granulomas (Hematoxylin-Eosin, X70).

involvement ${ }^{5,6}$ but the RV can be predominantly affected, as the authors have illustrated. Often cardiac sarcoidosis is clinically silent but it may result in bundle branch block or AV block due to involvement of the basilar septum.

Cardiac death is common. ${ }^{7}$ Sudden, presumably arrhythmic death has been reported in 7 of 43 patients diagnosed with cardiac sarcoidosis. ${ }^{8}$ It also may present with valvular regurgitation as a result of papillary muscle involvement or progressive heart failure. VT can be seen in ARVC or $\mathrm{ARVD}^{9}$ and in cardiac sarcoidosis. ${ }^{10,11}$ In one autopsied case, both cardiac sarcoid and ARVC or ARVD coexisted in the same patient. ${ }^{12}$ When there is VT associated with focal or global RV abnormalities, this may result in diagnostic confusion especially if there is no systemic evidence of sarcoidosis. Cardiac biopsies are frequently inconclusive since both disease processes tend to be focal and can easily be missed. Angiography or echocardiography of the heart delineates regional structural (aneurysm, thinning) and/or functional (hypokinesis, dyskinesis, perfusion defects) abnormalities without specifying the underlying disease process. Extensive septal involvement would favor the di- agnosis of myocardial sarcoidosis though ARVC or ARVD may affect the septum and the left ventricular free wall. ${ }^{13,14}$

Once cardiac sarcoidosis is diagnosed or suspected, therapy with corticosteroids is usually recommended. There is a report indicating that steroid treatment may be effective in the treatment of VT due to sarcoidosis. ${ }^{15}$ This is a practical reason for attempting to diagnose sarcoidosis since the VT may be responsive to steroids where it would not be expected to be successfully treated by this means in patients with ARVC or ARVD. If cardiac transplantation is considered, there should be awareness that there can be recurrences of sarcoidosis in the allograft. ${ }^{16}$

\section{Summary}

These case reports highlight the need to consider cardiac sarcoidosis in the differential diagnosis in patients who present with VT and RV abnormalities and are suspected of having ARVC or ARVD. Systemic symptoms, in particular pulmonary findings, septal involvement, or AV conduction disturbance, should raise the possibility of cardiac sarcoidosis. 


\section{Addendum}

A 41 year old man presented with near syncope and ventricular tachycardia. The resting electrocardiogram showed sinus rhythm with first degree AV block (PR $230 \mathrm{~ms}$ ). The QRS duration was increased (130 ms) and had a right bundle branch block pattern with negative $\mathrm{T}$ waves in lead V1 V3. In addition, low amplitude low frequency potentials were seen in lead V1 - V3 suggestive of epsilon waves. During electrophysiologic evaluation monomorphic ventricular tachycardia was easily induced (two morphologies both with left bundle branch block pattern). Evaluation by echocardiography showed normal LV size and function, normal valvular structures and mild to moderate RV dilatation. An RV angiogram revealed a focal area of inferior akinesis and increased RV trabeculation compatible with arrhythmogenic right ventricular dysplasia. Myocardial biopsies were normal and

\section{References}

1. Corrado D, Fontaine G, Marcus FI, et al. Arrhythmogenic right ventricular dysplasia/cardiomyopathy: The need for an International Registry. Circulation 2000; 101:e101-e106 and J Cardiovasc Electrophysiol 2000; II:827-832.

2. Gendel BR, Young JM, Geriner DJ. Sarcoidosis: A review with twenty-four additional cases. Am J Med 1952; 12:205218.

3. Porter GH. Sarcoid heart disease. N Engl J Med 1960; 263:13501357.

4. Perry A, Vuitch F. Causes of death in patients with sarcoidosis: A morphologic study of 38 autopsies with clinicopathologic correlations. Arch Pathol Lab Med 1995; 119:167-172.

5. Valantine H, McKenna WJ, Nihoyannopoulous P, et al. Sarcoidosis: A pattern of clinical morphological presentation. Br Heart J 1987; 57:256-263.

6. Roberts WC, McAllister HA, Ferrans VJ. Sarcoidosis of the heart A clinicopathologic study of 35 necropsy patients (group I) and review of 78 previously described necropsy patients (group II). Am J Med 1977; 83:86-108.

7. Sharma OM P. Cardiac and neurologic dysfunction in sarcoidosis. Clin Chest Med 1997; 18:813-825.

8. Johnson C, Michele TM. The clinical management of sarcoidosis: A 50 year experience at the Johns Hopkins Hospital. Medicine 1999; 78:65-111. an MRI found no evidence of myocardial thinning or fat infiltration. The incidental finding of a small lung mass was made. Chest CT showed scarring of the right apex and two small mediastinal lymph nodes.

A biopsy of a chronically enlarged lymph node in the neck revealed: "replacement of most of the normal lymphoid cells with multiple noncaseating granulomas compatible with sarcoidosis. Stains for mycobacterium and fungal organisms were negative."

\section{Comment}

The clinical presentation and initial laboratory evaluation was compatible with arrhythmogenic right ventricular dysplasia. However, biopsy proved sarcoidosis of the lymph node and pulmonary disease strongly suggests cardiac sarcoidosis as the cause of this patient's cardiac disease.

9. Marcus FI, Fontaine G. Arrhythmogenic right ventricular dysplasia/cardiomyopathy. A Review. PACE 1995; 18:1298-1314.

10. Winters SL, Cohen M, Greenberg S, et al. Sustained ventricular tachycardia associated with sarcoidosis: Assessment of the underlying cardiac anatomy and the prospective utility of programmed ventricular stimulation, drug therapy and an implantable antitachycardia device. J Am Coll Cardiol 1991; 18:937-943.

11. Wilkins CE, Barron T, Lowrimore G, et al. Cardiac sarcoidosis: Two cases with ventricular tachycardia and review of cardiac involvement in sarcoid. Tex Heart Inst J 1985; 12:377-383.

12. Scully RE, Mark EJ, McNeely WF, et al. Case records of the Massachusetts General Hospital. N Engl J Med 1996; 335:1378-1386.

13. Yazaki Y, Isobe M, Hiramitsu S, et al. Comparison of clinical features and prognosis of cardiac sarcoidosis and idiopathic dilated cardiomyopathy. Am J Cardiol 1998; 82:537-540.

14. Bharati S, Levi M, Denes P, et al. Infiltrative cardiomyopathy with conduction disease and ventricular dysrhythmia: Electrophysiological and pathological correlations. Am J Cardiol 1980; 45:163173.

15. Tarantini G, Menti L, Angelini A, et al. Life-threatening ventricular arrhythmias associated with giant cell myocarditis (possibly sarcoidosis). Am J Cardiol 2000; 85:1280-1282.

16. Barbers RG. Role of transplantation (lung, liver and heart) in sarcoidosis. Clin Chest Med 1997; 8:865-874. 